University of Nebraska - Lincoln

DigitalCommons@University of Nebraska - Lincoln

2012

Variations of the apparent angular size of the Sun across the entire Solar System: Implications for planetary opposition surges

Estelle Déau

NASA Jet Propulsion Laboratory, Pasadena CA

Follow this and additional works at: https://digitalcommons.unl.edu/nasapub

Part of the Physical Sciences and Mathematics Commons

Déau, Estelle, "Variations of the apparent angular size of the Sun across the entire Solar System: Implications for planetary opposition surges" (2012). NASA Publications. 49.

https://digitalcommons.unl.edu/nasapub/49

This Article is brought to you for free and open access by the National Aeronautics and Space Administration at DigitalCommons@University of Nebraska - Lincoln. It has been accepted for inclusion in NASA Publications by an authorized administrator of DigitalCommons@University of Nebraska - Lincoln. 


\title{
Variations of the apparent angular size of the Sun across the entire Solar System: Implications for planetary opposition surges
}

\author{
Estelle Déau* \\ Jet Propulsion Laboratory, NASA/Caltech, 4800 Oak Grove Drive, Pasadena, CA 91109, USA
}

A R T I C L E I N F O

\section{Article history:}

Received 11 January 2011

Received in revised form

20 February 2012

Accepted 20 February 2012

Available online 13 March 2012

\section{Keywords:}

Planetary surfaces

Phase curves

Opposition effect

Finite size of the Sun

Convolution

Deconvolution

\begin{abstract}
A B S T R A C T
We test several convolution and deconvolution models on phase curves at small phase angles $\left(0.001^{\circ}<\alpha<1.5^{\circ}\right)$ that have the highest phase angle sampling to date. These curves were provided by cameras onboard several NASA missions (Clementine/UVVIS, Galileo/SSI and Cassini/ISS) when the Sun had different apparent angular radii $\left(\alpha_{\odot}=0.266^{\circ}, 0.051^{\circ}, 0.028^{\circ}\right)$. For the smallest phase angles, the brightness of the objects (Moon, Europa and the Saturn's rings) exhibits a strong round-off below the angular size of the Sun. The brightness continues to increase below $\alpha_{\odot}$ before finally flattening at $0.4 \alpha_{\odot}$. These behaviors are consistent with the convolution models tested. A simple deconvolution model is also used and agrees with laboratory measurements at extremely small phase angles that do not show any flattening [Psarev V, Ovcharenko A, Shkuratov YG, Belskaya I, Videen G. Photometry of particulate surfaces at extremely small phase angles. J Quant Spectrosc Radiat Transfer 2007;106:455-63].
\end{abstract}

Published by Elsevier Ltd.

\section{Introduction}

Brightness variations of a surface are often related to the phase angle (the angular separation between the observer and the light source seen by the surface). When the observer and the light source are aligned, with the phase angle close to $0^{\circ}$, a strong surge in the brightness called the opposition effect is observed [1]. The opposition effect is observed in the phase curves of a wide range of astronomical objects [2-14] for phase angles up to $20^{\circ}$. Although observed since the 19 th century $[15,16]$, the opposition effect is not well understood [4].

Several theoretical models explain the shape of the surge by different mechanisms.

(1) The shadowing mechanism [15] consists in the progressive disappearing of mutual shadows of regolith grains [17-21]. A similar effect, caused by the surface

\footnotetext{
* Corresponding author. Tel.: +1 818393 1014;

fax: +18183934495

E-mail address: estelle.deau@jpl.nasa.gov
}

roughness at high scale (or macroscopic roughness), is called shadow hiding mechanism, and also plays a role on the opposition effect surge [18,22-24]. By taking into account the influence of hierarchical surface structure, intermediate cases between shadowing and shadow hiding mechanisms have been investigated by several authors [25-28].

(2) The coherent backscattering mechanism or weak localization of photons [29-32], which is a more recent theory than the shadowing and shadow hiding mechanisms. The coherent backscattering mechanism is caused by waves propagating in a reversed path through a medium, that interfere constructively in the backward direction but not necessarily in other directions, making it preponderant for the smallest phase angles [29]. The coherent backscattering mechanism originates from the multiple reflections inside the medium. Those reflections are composed of an incoherent contribution from: the scattered background radiation, and a coherent interferential contribution in the exact backscattering direction [32]. This mechanism, via the incoherent multiple scattering, has been suggested to weaken the 
shadow effects, by decreasing the opposition surge [33-35]. The first considerations of the coherent backscattering effect in the planetary context was suggested in $[29,36-39]$.

(3) The near-field mechanism [40-42] consists of interferences between scatterers in the near field and is caused by the inhomogeneity of waves in the immediate vicinity of constituent particles.

(4) The internal-field mechanism $[43,44]$ is caused by the longitudinal and transverse components of the internal electric fields induced in single wavelength-scale scatterers. This mechanism can be one among many others that generate the single-particle scattering indicatrix. The indicatrix has a backscatter component that may contribute to the backscatter of particulate (regolith-like) surfaces. Experimental attempts to separate single and multiple scattering effects on backscattering have been made in [45-47].

The contribution of these four mechanisms in the opposition surge is still a matter of debate [48-54]. Also, the size of the Sun (as seen by the observed surface) influences the opposition effect $[18,55,56]$. Since the Sun has a non-zero angular size, none of those four mechanisms can be applied as-is. The size of the Sun results on a flattening of the phase curves. This flattening was first seen on the Moon in Apollo 8 photographs [2,3]. It was absent in early measurements from Clementine images [57], but [58] put their analysis into question. Later on, reanalysis of the same Clementine data exhibited a flattening [7] see their Fig. 10.

Refs. [18,55,56,5] have studied the correlation between the angular size of the Sun and the flattening of the phase curve near $0^{\circ}$. From a mathematical point of view, the flattening of a phase curve corresponds to the convolution of "intrinsic" phase curve of the surface by the limb darkening function of the Sun [59,60]. The opposition surge will be broader when the solar angular radius is large (e.g. on the Moon), and narrower when the solar angular radius is small (e.g. on neptunian satellites and Kuiper belt objects). As a result, it is widely accepted that the size of the Sun has a major role in flattening the phase curve of the Moon [5], but the flattening is more often considered as negligible - or not considered at all - for bodies in the outer Solar System [9,61]. It is acceptable to neglect the solar size bias when considering an isolated phase curve; however, it becomes a concern when comparing Solar System objects at different distances from the Sun (and therefore, at different angular solar size). Refs. [56,14] have demonstrated that the amplitude of the opposition surge, as well as its angular width, are a function of the distance from the Sun; and that this result applies when considering objects both from the inner and outer Solar System. Acknowledging those results, we decide to address two questions:

(1) quantifying the influence of the angular size of the Sun on phase curves of planetary surfaces across the Solar System;

(2) determining the shape of the "intrinsic" phase curve of those planetary surfaces, if the Sun were a pointlike source.
These two questions can be treated in terms of convolution (adding the effect of the angular size of the Sun to a point model) and deconvolution (processing real data to remove the effect of the Solar angular size).

The present paper aims at studying the convolution and the deconvolution methods. In Section 2, we present our dataset: it has a high sampling in phase angle, and presents a flattening. Then, we perform a convolution with a morphological (non-physical) phase function to fit the phase curves. In Section 3, we use a deconvolution model and discuss the variation of the brightness below the angular size of the Sun, i.e. if the Sun was seen as a punctual source. In Section 4, we present the physical implications of the convolution model and we show the consequences of the deconvolution on the opposition surge of Solar System objects. Section 5 presents the limitations of our method. We summarize our conclusions in Section 6.

\section{The convolution problem}

\subsection{Convolved data}

To deal correctly with the convolution problem, we need phase curves at small phase angles and with a high sampling rate for those small phase angles. The phase curves presented here have a lot of points below the angular size of the Sun, and are therefore satisfactory. They were obtained for objects with different albedos: $\varpi_{0}=0.21$ for the Moon's disk [62], $\varpi_{0}=0.96$ for Europa's disk [63], $\varpi_{0}=0.75$ for the A ring, $\varpi_{0}=0.90$ for the B ring, $\varpi_{0}=0.28$ for the $C$ ring, $\varpi_{0}=0.35$ for the Cassini Division [64].

The opposition data of the Moon, Europa and Saturn's rings consist in the brightness from a few tenths of degrees down to angular size of the Sun. For consistency, we keep using the scale of the brightness from the original works of $[7,6,65]$.

In Fig. 1, the phase curves are displayed in both linear and logarithmic scale of phase angle. This is particularly relevant for appreciating the high phase angle sampling of these phase curves.

The Moon, Europa and the Saturn's rings phase curves offer us the opportunity to observe the effect of the nonzero size of the Sun at different scales. To compute the apparent angular radius of the Sun for a given body, we use the following expression:

$\alpha_{\odot}=\arcsin \frac{R_{\odot}}{D_{\text {body }-\odot}}$

where $R_{\odot}$ is the radius of the Sun $\left(6.955 \times 10^{8} \mathrm{~m}\right.$, see [66]) and $D_{\text {body-} \odot}$ is the distance from the object to the Sun.

Using Eq. (1) at the epoch of the Clementine, Galileo and Cassini data, the Sun had respectively an angular radius of about $\alpha_{\odot}=0.266^{\circ}, 0.051^{\circ}$ and $0.028^{\circ}$. As a result we can observe a ratio of 10 between the apparent solar size seen from the Moon and the one seen from Saturn (see Fig. 1).

Moon phase curves for the blue filter $(\lambda=415 \mathrm{~nm})$ and the red filter $(\lambda=750 \mathrm{~nm})$ were obtained from Clementine photographs in the vicinity of crater Blagg by [7]. The crater Blagg $\left(1.3^{\circ} \mathrm{N}, 1.5^{\circ} \mathrm{E}\right)$, intensively observed by the 

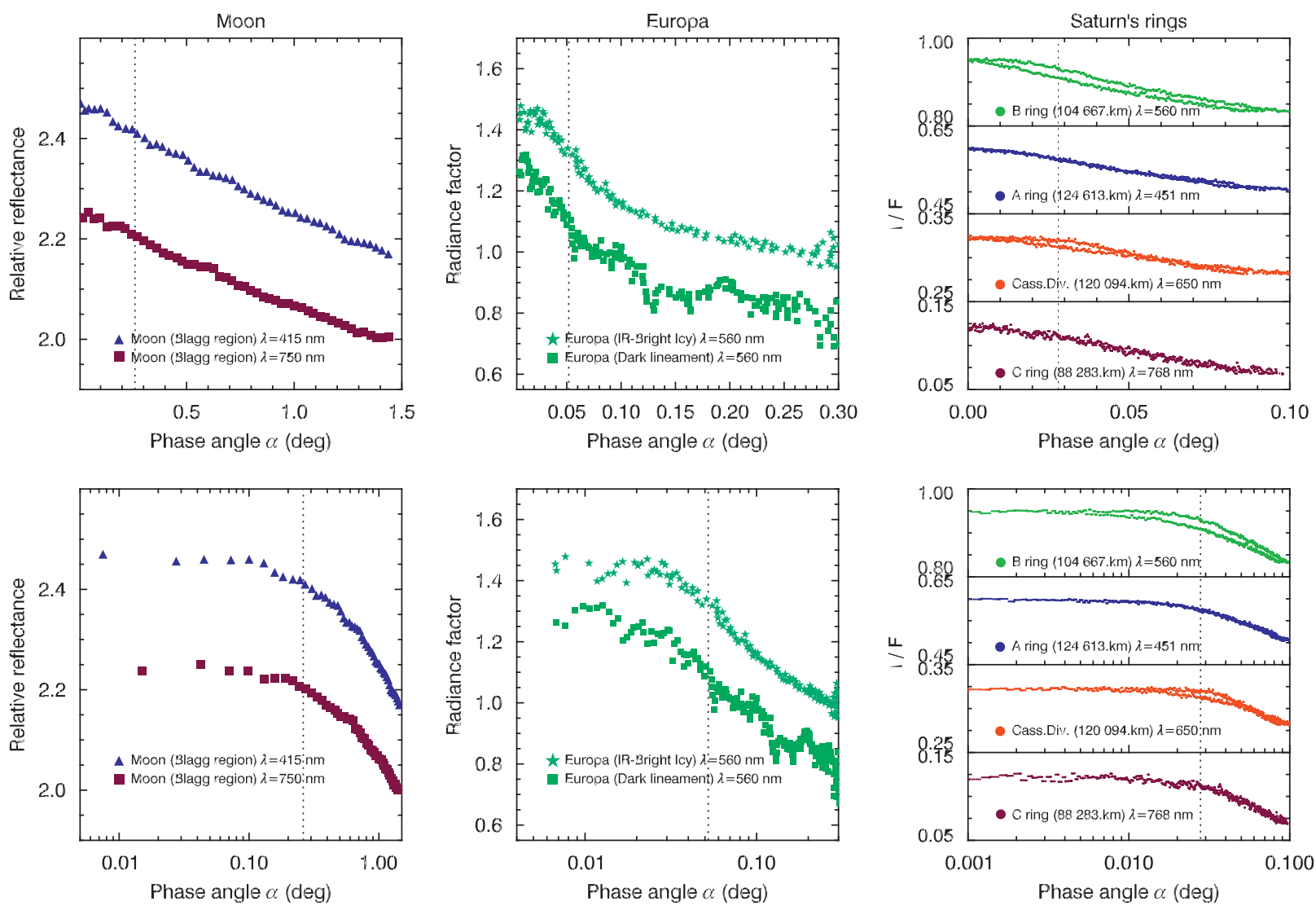

Fig. 1. Phase curves of the Moon (left panels), Europa (center panels) and the Saturn's rings (right panels) from images of Clementine, Galileo and Cassini. Vertical dotted lines correspond to solar angular radii $\left(\alpha_{\odot}\right)$. Top panels correspond to the phase curves with a linear scale of phase angle. Bottom panels correspond to the same phase curves but represented with a logarithmic scale of phase angle.

Clementine mission is tiny, circular and is located on the Sinus Medii. Ref. [7] averaged the pixels of different images that have the same phase angles, excluding crater walls and tilted areas.

Europa phase curves were obtained from Galileo green images $(\lambda=560 \mathrm{~nm})$ of two regions called by [6] "IRbright icy material" and "dark lineament material". The "IR-bright icy material" is analogous to [67]'s "infraredbright plains terrains" and is distinguishable by its higher albedo in the near-infrared $(1 \mu \mathrm{m})$. The "dark lineament material" corresponds to dark curvilinear features that cover Europa's surface, such as dark bands and dark portions of triple bands. The phase curves are a little bit noisy - and noisier than the Clementine phase curves - so they have been binned and averaged in $0.001^{\circ}$ increments of phase angle.

Saturn's rings phase curves were obtained from Cassini images from the Narrow Angle Camera (NAC) of ISS at different distances from the Saturn center [65]. To retain the specific behavior of the different areas (A, B, C rings, and Cassini Division), the data was not averaged. The phase curves shown here correspond to small regions in the rings $(<40 \mathrm{~km})$ that have very low variations of optical depth within them [65]. Each curve was extracted from a single image that shows the opposition spot. For diversity, we choose images with different filters: $\lambda=$ $560 \mathrm{~nm}$ for the $B$ ring, $\lambda=451 \mathrm{~nm}$ for the A ring, $\lambda=$ $650 \mathrm{~nm}$ for the Cassini Division and $\lambda=768 \mathrm{~nm}$ for the $C$ ring. In Fig. 1, it is possible to see two plots for each phase curve. They correspond to two different viewing geometries (typically a variation of a few degrees of the emergence angle). These two curves have been also observed by VIMS (see [68, Fig. 4]) and interpreted as an effect of the coherent backscattering [69].

When looking at the phase curves in linear scale of phase angle, the flattening near $0^{\circ}$ of phase angle is not obvious (top panel of Fig. 1). However, when displaying the data in logarithmic scale of phase angle (bottom panel of Fig. 1), we observe the following:

- for phase angles larger than the angular radius of the Sun, the brightness decreases with increasing phase angle;

- but for phase angles smaller than angular radius of the Sun, the brightness remains constant whatever the phase angle.

Actually, the flattening cut-off does not appear exactly at the angular radius of the Sun, but slightly below-and this is the case for all the observed phase curves. The 
flattening seems to be linked with the solar angular size; and we will see later why the cut-off does not happen exactly at the solar angular radius. Also, the flattening on logarithmic scale is not only due to the angular size of the Sun. It may be observed over a wide range of phase angles, see [70].

\subsection{Convolution models}

We will now fit the opposition surge data with a morphological function. But most morphological functions assume that the Sun is a point light source. To acknowledge the fact that the Sun is neither a point light source, nor a perfectly uniform disc, we will convolve the morphological function with a solar limb darkening function.

For the morphological function, we choose the linearexponential function of [71] because it was widely used to parameterize planetary opposition phase curves [64,14, 72,73]. Its mathematical expression is

$r_{\text {linexp }}(\alpha)=I_{p} \cdot \exp \left(\frac{-\alpha}{2 w}\right)+I_{b}+I_{s} \cdot \alpha$

where the intensity of the peak is $I_{p}$, the intensity of the background is $I_{b}$, the slope of the linear part is $I_{s}$ and the angular width of the peak is $w$.

The limb darkening functions are functions depending on the usual photometric coordinate system. Let us introduce $\theta_{e}=\arccos \mu$, the polar angle with respect to the outward normal of the surface and $\phi_{e}$, the corresponding azimuth measured from the incidence plane in such way that the notation $\Omega_{e}=\left(\theta_{e}, \phi_{e}\right)$ specifies a particular direction. For example, we will assume the Sun symmetric about the angular direction $\delta_{\odot}=$ $\left(\theta_{\odot}, \phi_{\odot}\right)$ of its midpoint, $\left(\phi_{\odot}=0^{\circ}\right)$; and the solar radiation is considered incident only in the direction $\Omega_{i}=\left(\theta_{i}, \phi_{i}\right)$. For convenience, we take $\theta_{i}=\arccos \mu_{i}$. The phase angle can be described by the four angles $\theta_{e}, \theta_{i}, \phi_{e}$ and $\phi_{i}$ :

$\cos \alpha=\cos \theta_{e} \cos \theta_{i}+\sin \theta_{e} \sin \theta_{i} \cdot \cos \left(\phi_{e}-\phi_{i}\right)$

As a result, our analysis is restricted to the Cassini/ISS data of the Saturn's rings since we do not have the values of the coordinates angles for the data of the Moon and Europa. It would be possible to approximate the geometry at the epoch of the observations of Clementine and Galileo (using the JPL Solar System Ephemeris system, available at http://ssd.jpl.nasa.gov/?horizons, see the results of [74]), but not in a satisfactory manner for the exact locations of the surface chosen by [6,7].

There are many limb darkening models [75]. We test here two models that have been used previously for the Saturn's rings opposition effect [55,72]. The first one is the solar limb darkening function of [59]:

$W\left(\mu^{\prime}\right)=a_{\lambda}+b_{\lambda} \mu^{\prime}+c_{\lambda}\left[1-\mu^{\prime} \cdot \log \left(1+\frac{1}{\mu^{\prime}}\right)\right]$

where $\mu^{\prime}=\cos \theta^{\prime}$ and $\theta^{\prime}$ varies from $0^{\circ}$ to the Sun's apparent angular radius $\alpha_{\odot} . a_{\lambda}, b_{\lambda}$ and $c_{\lambda}$ are coefficients that depend on the wavelength (see Table 1).
Table 1

Wavelength-dependent parameters of solar limb darkening functions of $[59,60]$.

\begin{tabular}{|c|c|c|c|c|c|c|}
\hline \multirow[t]{2}{*}{$\lambda_{o b s}(\mathrm{~nm})$} & \multicolumn{4}{|c|}{ Model [59] } & \multicolumn{2}{|c|}{ Model [60] } \\
\hline & $\lambda(\mathrm{nm})$ & $a_{\lambda}$ & $b_{\lambda}$ & $c_{\lambda}$ & $\lambda(\mathrm{nm})$ & $\beta_{\lambda}$ \\
\hline 451 & 440 & 0.49375 & 0.62584 & -0.38974 & 454.355 & 0.633 \\
\hline 568 & 560 & 0.75079 & 0.41593 & -0.54334 & 559.950 & 0.502 \\
\hline 650 & 660 & 0.81999 & 0.34918 & -0.55132 & 649.250 & 0.413 \\
\hline 752 & 750 & 0.90904 & 0.26588 & -0.57006 & 770.820 & 0.342 \\
\hline
\end{tabular}

The convolution of the linear-exponential function of [71] to the limb darkening function is

$r_{1}=\frac{\int_{0}^{\alpha_{\odot}} d \theta^{\prime} \int_{0}^{2 \pi} r_{\text {linexp }}\left(\Omega^{\prime}\right) \cdot W\left(\cos \theta^{\prime}\right) \sin \theta^{\prime} \cos \theta_{i}\left(\delta^{\prime}\right) d \phi^{\prime}}{\int_{0}^{\alpha_{\odot}} d \theta^{\prime} \int_{0}^{2 \pi} W\left(\cos \theta^{\prime}\right) \sin \theta^{\prime} \cos \theta_{i}\left(\delta^{\prime}\right) d \phi^{\prime}}$

where the direction $\delta^{\prime}$ is given by the coordinates $\left(\theta^{\prime}, \phi^{\prime}\right)$, $\cos \theta_{i}=\cos \theta^{\prime} \cos \theta_{\odot}-\sin \theta^{\prime} \sin \theta_{\odot} \cdot \cos \phi^{\prime}$ and $\sin \phi_{i}=$ $\sin \theta^{\prime} \sin \phi^{\prime} / \sin \theta_{i}$.

The second limb darkening function tested here is the one-parameter solar limb darkening model, well detailed in [60]:

$I_{\lambda}(\hat{r})=\left(1-\hat{r}^{2}\right)^{\beta_{\lambda}}$

where $I_{\lambda}(\hat{r})$ is the limb-darkened solar intensity, $\hat{r}$ is the normalized radial coordinate of the solar disk, and $\beta_{\lambda}$ is a wavelength-dependent constant (see Table 1 ). We convolved the linear-exponential function to this function by doing:

$r_{2}=\frac{\int r_{\text {linexp }}\left(\Omega^{\prime}\right) I_{\lambda}\left(\Omega^{\prime}\right) d \Omega^{\prime}}{\int I_{\lambda}\left(\Omega^{\prime}\right) d \Omega^{\prime}}$

where $I_{\lambda}\left(\Omega^{\prime}\right)$ is the limb-darkened solar intensity, and the integrations were made over the solid angle $d \Omega^{\prime}=\sin \theta^{\prime} d \theta^{\prime} d \phi^{\prime}$ with the boundaries $0 \leq \phi^{\prime} \leq 2 \pi$ and $0 \leq \theta^{\prime} \leq \alpha_{\odot}$.

In Fig. 2, we represent both best fits of the convolutions models $r_{1}(\alpha)$ and $r_{2}(\alpha)$ and found that they are quite identical. The convolved linear-exponential functions fit very well the Cassini data. As a result, both convolution models tested here give a good agreement with the data and between themselves, as shown in Fig. 2. The flattening of the phase curves is progressive and effective at approximately $0.4 \alpha_{\odot}$. This value is found by looking at the derivative of the convolved linear-exponential function fit to the data.

It is interesting to note that for small phase angles, the best fits from the convolved functions are quite identical to the best fit of the linear-exponential function itself. Because Eq. (2) is almost linear at small phase angles, as a consequence the convolution almost automatically yields the same result.

However, when including data for wider ranges of phase angles, we will see a significant difference. The linear-exponential function will not fit as well; but the convolved function will remain accurate. 


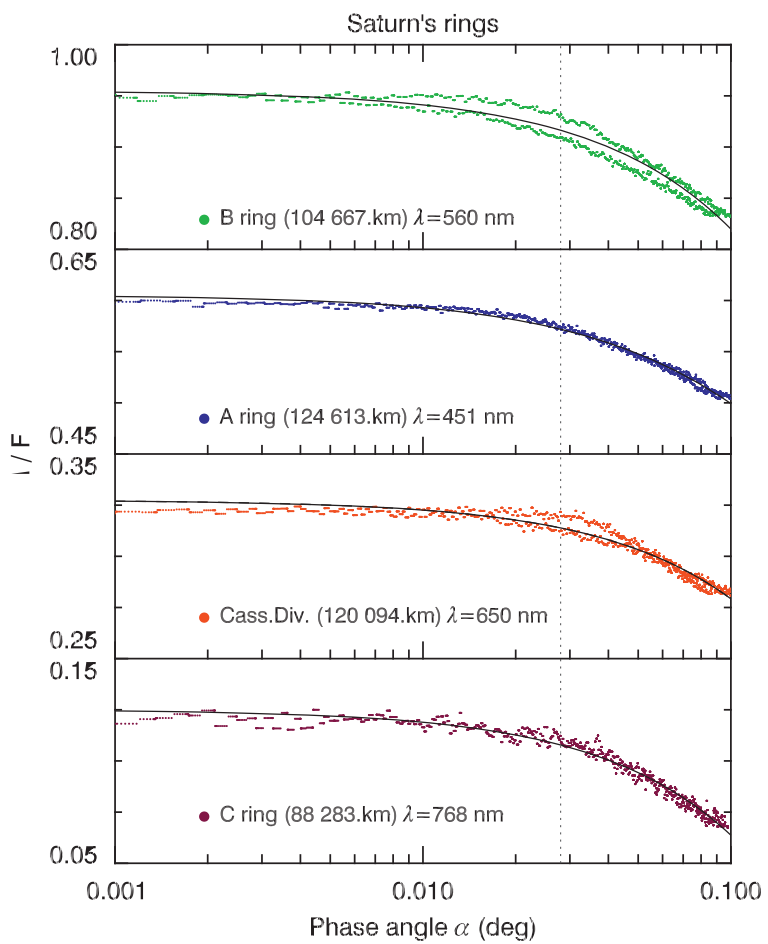

Fig. 2. Phase curves of the Saturn's rings fit with the model of [59] in solid curves and the model of [60] in dotted curves. The vertical dotted line corresponds to solar angular radius $\left(\alpha_{\odot}\right)$ at Saturn.

\section{The deconvolution problem}

We established the validity of the convolution of the linear-exponential function with several limb darkening functions. We will now deconvolve the data, in order to obtain information about the "intrinsic" behavior of phase curves-i.e. their behavior if the Sun was a punctual source. As stated above, the linear-exponential function intrinsically flattens as $\alpha \rightarrow 0^{\circ}$, see [14]. Therefore, the goal of the deconvolution method is to study the phase curves "intrinsic" behavior over wider phase angle ranges.

A deconvolution method is particularly challenging because it should remove two effects which are not perfectly known: an observational effect $[55,56,18,5,14]$ and a physical effect [76,77]. Because of that, the deconvolution is not commonly used on phase curves.

Assimilating the Sun to a point light source is not only a theoretical artefact. In the next section, we will introduce laboratory experiments confirming this reasoning.

\subsection{Deconvolved data}

A recent paper of [78] provided phase curves of particulate surfaces at extremely small phase angles $\left(0.008-1.51^{\circ}\right)$. Surprisingly, they found that their data do not exhibit any flattening as the phase angle approaches zero. Indeed, as shown in Fig. 3, when representing several phase curves observed by [78] in logarithmic scale, it can be clearly seen that the brightness increases without round-off.
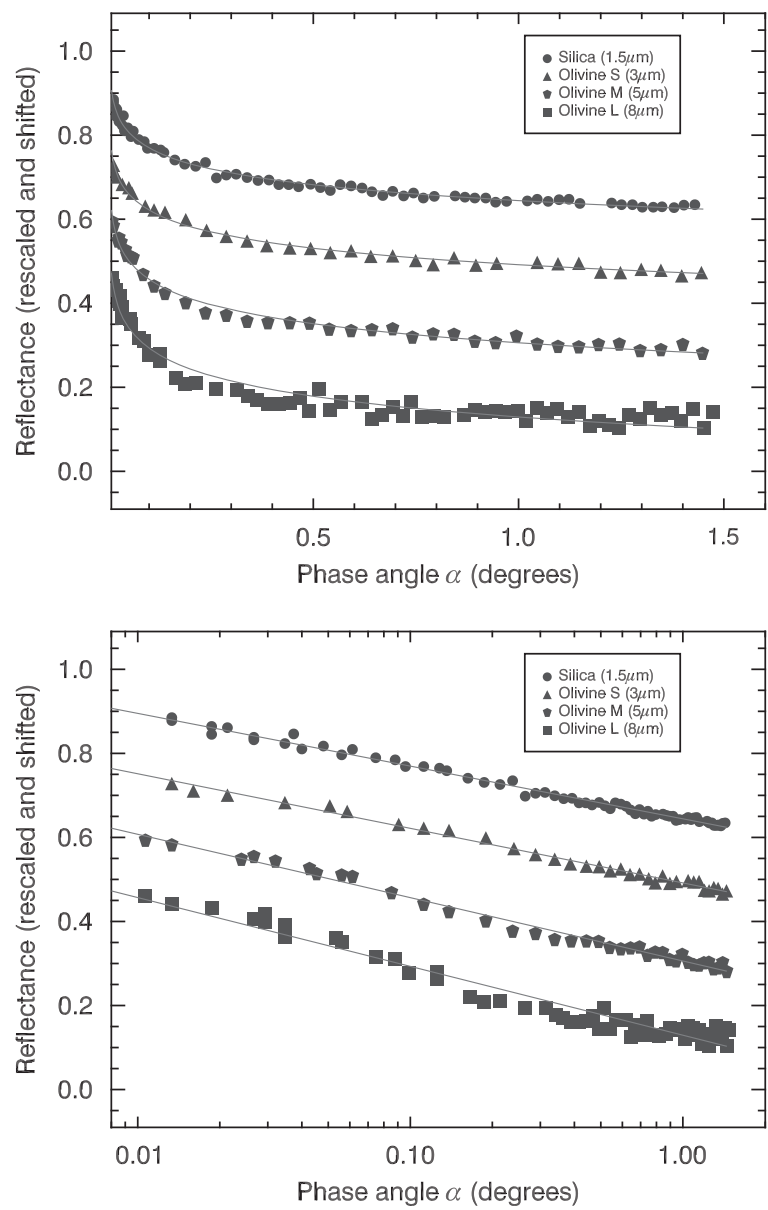

Fig. 3. Laboratory phase curves of [78] at very small phase angles in linear scale of phase angle (top panel) and in logarithmic scale of phase angle (bottom panel). Solid curves represent the best fit with the deconvolution model of [14].

However, to reach the smallest phase angles, [78] had to decrease the light source (laser) aperture and increase laser-sample separation distance from $25 \mathrm{~m}$ to $40 \mathrm{~m}$. A reduced aperture and an increased distance translate into a smaller angular size of the light source. Therefore, as they were doing measurements for smaller and smaller phase angles, [78] also made the light source more and more point-like. Their protocol had indeed the effect of a "experimental" deconvolution.

Because each point of their phase curves was acquired at a different angular size of the light source, the phase curves of [78] are intrinsically very different from other laboratory phase curves [79-89] where the angular size of the light source is constant.

Nevertheless, the origin of the non-flattening of [78]'s phase curves can be interpreted differently. Recently, [90] proposed that the shadowing could influence the coherent backscattering by blocking its reciprocal components, in this way, the resulting phase curves could not show any flattening. The authors of that paper explained with this mechanism why all the laboratory curves (and particularly that of $91,86,78]$ ) do not present any flattening. 

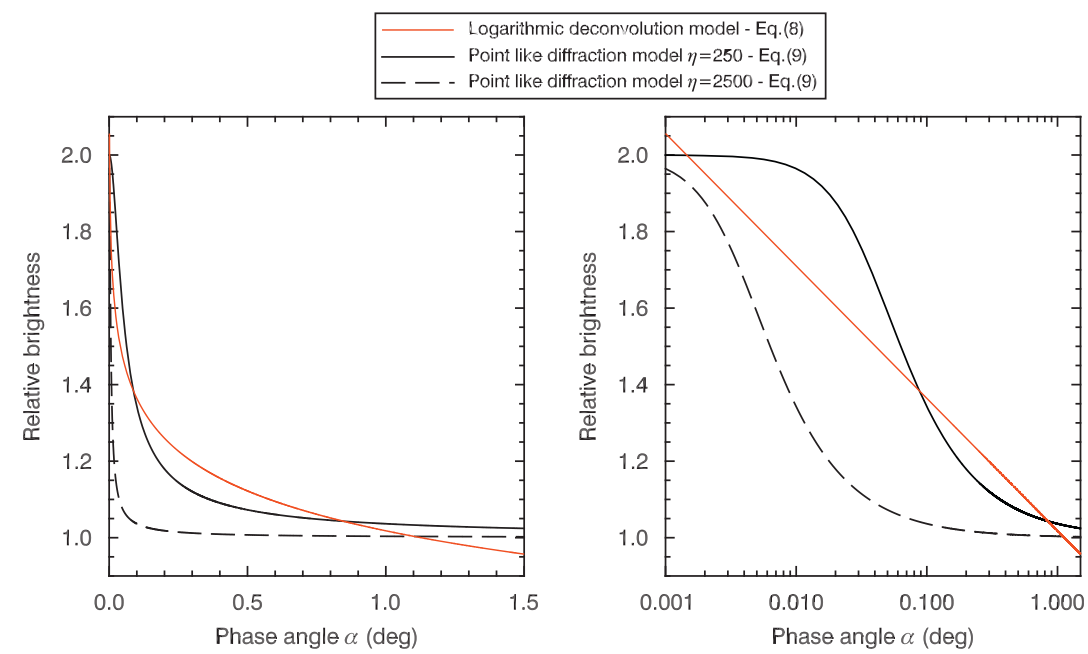

Fig. 4. Comparison of the phase curves of the deconvolution model of [14], with $a_{0}=1.018$ and $a_{1}=-0.15$ and with the diffraction model of [56], with $\eta=250$ and $\eta=2500$, that assumes a point light source. The phase curves are displayed in linear scale of phase angle (left panel) and logarithmic scale of phase angle (right panel).

However, it is very hard for laboratory experiments using goniometers to measure angles less than the angular size of the light source when this angular size remains constant. Indeed, there is always a minimum phase angle that permits the unobstructed light beam to pass the detector and illuminate the sample. And this minimum phase angle $\left(\alpha_{\min }\right)$ is a often far greater than the angular radius of the light source $\left(\alpha_{\circledast}\right)$, for example:

- $\alpha_{\min }=2^{\circ}$ while $\alpha_{\circledast}=0.25^{\circ}$ when using a $20 \mathrm{~W}$ Tungsten halogen lamp [79];

- $\alpha_{\min }=0.05^{\circ}$ while $\alpha_{\circledast}=0.0074^{\circ}$ when using a $15 \mathrm{~mW}$ He-Ne laser [86]. ${ }^{1}$

Since most laboratory experiments use He-Ne laser $[80,81,84,86,87,89,92]$ and assuming that the distances from the beamsplitter to the detector vary from approximately from $0.5 \mathrm{~m}$ [87] to $2.31 \mathrm{~m}$ [81], we conclude that the maximum angular radius of the laser beam as seen by the sample never exceeded $0.086^{\circ}$ for these studies. Such a small phase angle is rarely measured, we believe this is why laboratory phase curves do not exhibit a round-off due to the finite size of the light source. Moreover, since the flattening is effective at $0.4 \alpha_{\circledast}$ (see Section 2.2) it is then impossible to observe a flattening with a laser beam as a light source when $\alpha_{\min }>0.4 \alpha_{\circledast}{ }^{2}$

\subsection{Deconvolution model}

The goal of the deconvolution model is to prevent the flattening of the phase curve at the phase angles corresponding to the apparent size of the light source. This

\footnotetext{
${ }^{1}$ With an assumed radius of $0.3 \mathrm{~mm}$ for the He-Ne laser beam used by [86] and a mirror-sample measured distance of $2.31 \mathrm{~m}$, see $[86,81]$ for details.

${ }^{2}$ Unfortunately, since we did not find any reference of the value of the angular radius of the light source used by [91], it was impossible to infer at which phase angle the flattening should occur.
}

model will then create a deconvolved phase curve or "intrinsic" phase curve, corresponding to the brightness behavior of the surface if the light source was a point.

Strictly speaking, the deconvolution of the problem is not straightforward because it is likely that the "intrinsic" phase curve should be already flattened by the coherent backscatter contribution to the opposition effect [76], otherwise the Maxwell equations would be violated [77]. This is why there are only a few models that deconvolve phase curves or at least consider the Sun as a point-like source [56]. So we use a simple model. The idea is to use a model that does not round-off below the solar angular size and that allows the same increase before and after the solar angular size without a slope change. This model is simply a logarithmic function of [93] and only this function cannot flatten at the smallest phase angles and can assume the same increase of the data below the light source's angular size, as for larger phase angles. This function was used as a deconvolution model by [14]

$r_{\log }(\alpha)=a_{0}+a_{1} \cdot \ln (\alpha)$

This assumption is consistent with the recent laboratory measurements of [78] at small phase angles (0.008$1.51^{\circ}$ ), that did not show any flattening of the phase curves, and that we consider to be deconvolved data in the previous section.

The deconvolution model could suggest a logarithmic trend for the brightness when $\alpha$ tends towards $0^{\circ}$ or on a logarithmic scale towards $10^{-n}$ with $n \rightarrow+\infty$. However, we did not assume an infinite logarithmic increase as required by Eq. (8) which would make impossible the computation of an amplitude for example. The deconvolution of the phase curves is assumed until $10^{-3}$ degree which is close to the angular size of the Sun as seen by the outer parts of the Solar System (see Table 2). For the larger phase angles, it can fit reasonably the data up to $20^{\circ}$, see [14].

In order to compare the deconvolution model of [14] with another similar model, we use the diffraction model of 

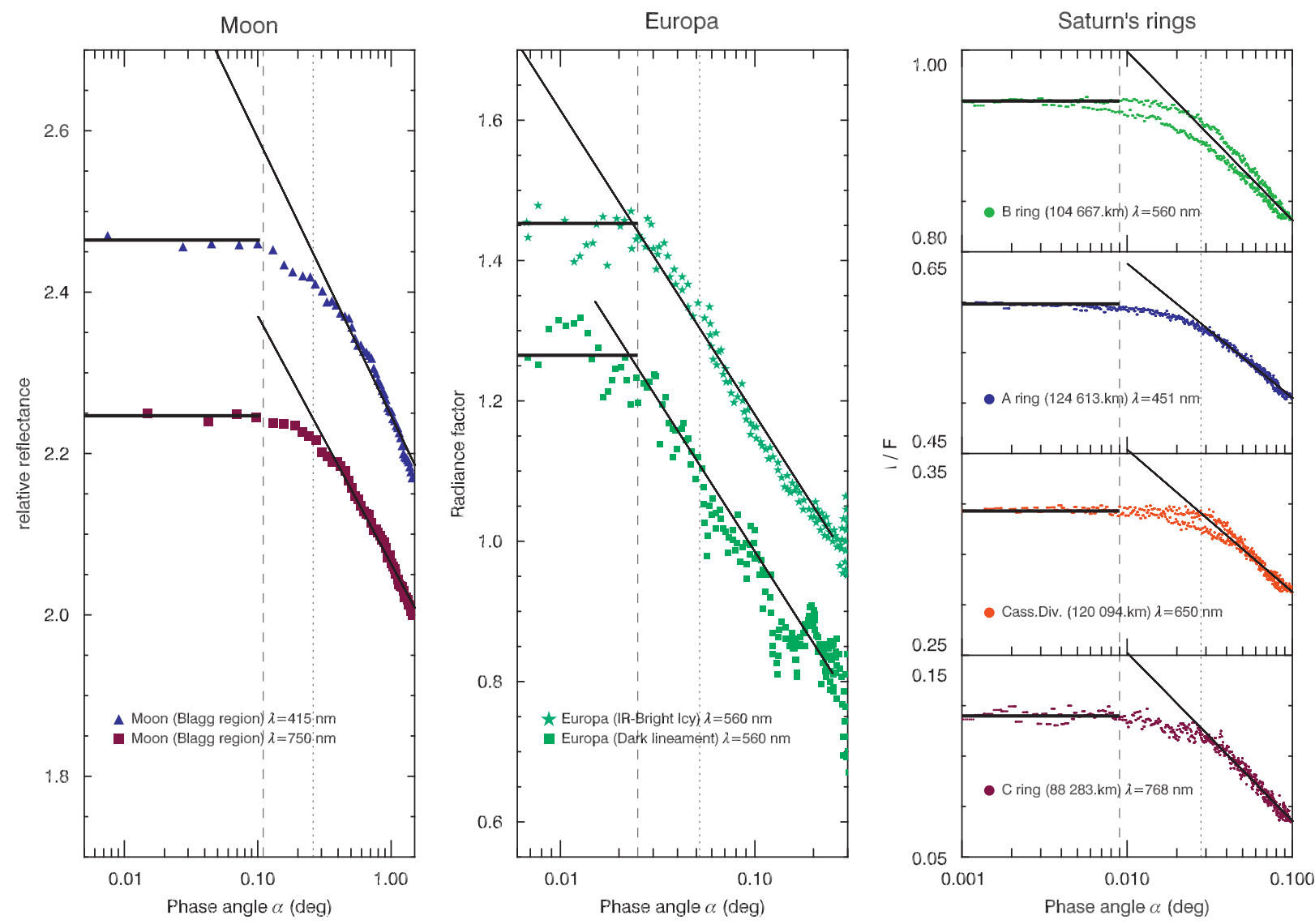

Fig. 5. Phase curves of the Moon (left panel), Europa (center panel) and the Saturn's rings (right panel) fit with the deconvolution model of [14] in solid lines. Vertical dotted lines correspond to the solar angular radius $\left(\alpha_{\odot}\right)$ and vertical dashed lines correspond to the effective flattening $\left(0.4 \alpha_{\odot}\right)$. Horizontal lines correspond to the flattened part of the phase curves data.

[56]. Since this model assumes that the light source is a point, we consider this model as a deconvolution model as well. This model describes the phase dependance of the brightness of a surface in the case of a point light source, within the framework of a diffraction model [36]:

$r_{\text {diffr }}(\alpha)=1+\frac{1}{\sqrt{1+\rho^{2} \alpha^{2}}}$

where $\rho=2 \pi \eta$, and $\eta$ is the characteristic radius of the light scattering region, referenced to the wavelength of light.

In Fig. 4, we compare the deconvolution model to the diffraction model. For the diffraction model, the value of $\eta$ is not clear when considering a point-like source, but for the Solar System, the $\eta$ values range is $13-1400$ according to [56], with 1400 corresponding to the value for the outer Solar System Objects. As a consequence we test two values: 250 and 2500 . We note that, whatever the $\eta$ values, the diffraction model flattens more at higher phase angle than at lower phase angle. In particular, the calculated brightness is constant for phase angles greater than $0.7^{\circ}$. As a result, the diffraction model is unable to reasonably fit the deconvolved data of [78]. For $\eta=2500$, the model would be unpractical for apparent solar angular radii less than $0.01^{\circ}$, i.e. for objects with a distance to the Sun greater than the Neptune's semi-major axis (see Table 2).
With the deconvolution model of [14], the behavior of the observed opposition surge appears to be more clear. The flattening of the observed phase curves is progressive and effective at approximately $0.4 \alpha_{\odot}$. This value is found by looking for the phase angle that corresponds to a flat behavior (Fig. 5) and is in agreement with the value found with the derivative of the convolved linear-exponential function (Section 2.2). In the case of Europa, the flattening does not seem progressive but is done at $0.4 \alpha_{\odot}$. This value is also the phase angle for which the deconvolution function does not fit anymore the phase curves while in the other phase curves-Saturn's rings and Moon, this angle is $\alpha_{\odot}$ (see Fig. 5). We believe this is due to the $0.001^{\circ}$ increment average of Europa's data performed by [6].

Finally, in the case of the Moon, although [7] emphasized that at the smallest phase angles, the average phase curves were not reliable, the strong flattening observed is similar to the that seen in Apollo 8 photographs ${ }^{3}[2,3]$.

To summarize, the deconvolution model of [14] offers a simple way to characterize the brightness below the solar angular radius. This deconvolution method naturally removes the flattening due to the non-zero size of the

\footnotetext{
${ }^{3}$ Disk-resolved observations of [2,3] from photographs were captured by an onboard Hasselblad Electric Camera (HEC) and reported by [5].
} 
light source, and the flattening caused by the coherent backscattering or any other physical mechanism. We believe this is why the data from [78] are only well fit by this deconvolution model (Fig. 3).

\section{Implications for the opposition effect in the entire Solar System}

\subsection{Convolution and deconvolution of a planetary dataset}

Solar System objects are a good opportunity to test the influence of the non-zero angular size of a light source. Previous works on the field, [94,56], were done without quantifying the effect of the solar angular size on the morphology of observed phase curves. In Fig. 6 are represented the convolved and deconvolved angular widths of the opposition surge as a function of the distance from the Sun (in Astronomical Units) for various Solar System objects (for details, see [14]).

The convolved HWHMs follow a power-law function with the heliocentric distance while the deconvolved HWHMs are independent of the distance from the Sun (see Fig. 6). With the deconvolved HWHMs, Solar System objects seem to be grouped by their albedo. This could be a consequence of the opposition effect mechanisms, since both shadow hiding and coherent backscattering are albedo-dependent [95,39].

\subsection{Convolution of a synthetic dataset}

While the work of $[94,14,56]$ demonstrated that the amplitude and the angular width of the opposition effect are correlated to the size of the Sun, there is still any work that quantifies the impact of the variation of the apparent size of the Sun across the entire Solar System. This is justified by the comparisons made by $[96,11]$ between the Moon and Mercury or the Moon, the Galilean satellites and Enceladus without taking into account the apparent size of the Sun seen by these surfaces.

In order to test the effect to the Solar angular radius in the Solar System, we compute the different apparent sizes $\alpha_{\odot}$ with Eq. (1) and the values of the distances in Table 2. As noted by [94], there is a factor 100 between the
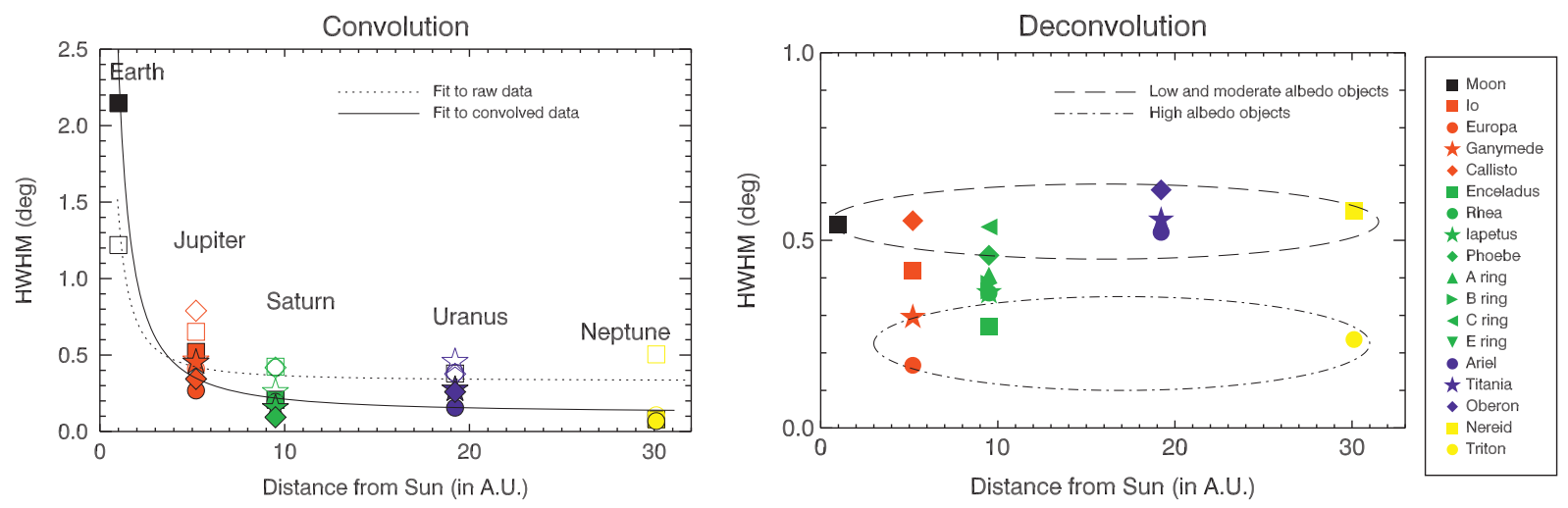

Fig. 6. Angular width of the opposition surge using the convolution method (left panel) and the deconvolution method (right panel) for the phase curve data of various rings and satellites of the Solar System from the study of [14].

Table 2

Geometric parameters for the observation of the opposition effect for each planet of our Solar System (minimum, maximum and averaged distances from the Sun refer to perihelion, aphelion and semi-major axis, and are in Astronomical Units from [66]. The apparent radius of the solar disk is in degrees and is calculated with Eq. (1) and the respective distances. Note that the Solar radius is $R_{\odot}=0.004649 \mathrm{AU}$ and $1 \mathrm{AU}=149.598 \times 10^{9} \mathrm{~m}$. Values for the main belt and Kuiper belt are added for completeness regarding the asteroids and their respective distances to the Sun correspond to their rough boundaries.

\begin{tabular}{|c|c|c|c|c|c|c|}
\hline \multirow[t]{2}{*}{ Primary } & \multicolumn{3}{|c|}{ Distance to Sun (AU) } & \multicolumn{3}{|c|}{ Angular solar radius $\left({ }^{\circ}\right)$} \\
\hline & $\min$. & $\max$ & mean & $\alpha_{\odot \min }$ & $\alpha_{\odot \max }$ & $\bar{\alpha}_{\odot}$ \\
\hline Mercury (ఫ) & 0.307 & 0.467 & 0.387 & 0.5703 & 0.8676 & 0.7087 \\
\hline Venus (q) & 0.718 & 0.728 & 0.723 & 0.3658 & 0.3709 & 0.3684 \\
\hline Earth (む) & 0.983 & 1.017 & 1.000 & 0.2619 & 0.2709 & 0.2664 \\
\hline Mars $\left(\sigma^{\top}\right)$ & 1.382 & 1.666 & 1.524 & 0.1598 & 0.1927 & 0.1758 \\
\hline Jupiter (4) & 4.951 & 5.455 & 5.203 & 0.0488 & 0.0538 & 0.0512 \\
\hline Saturn $(\hbar)$ & 9.014 & 10.044 & 9.529 & 0.0265 & 0.0295 & 0.0280 \\
\hline Uranus $(\hat{\odot})$ & 18.31 & 20.07 & 19.19 & 0.0132 & 0.0145 & 0.0139 \\
\hline Neptune $(\Psi)$ & 29.76 & 30.36 & 30.06 & 0.0087 & 0.0089 & 0.0088 \\
\hline Main belt (мв) & $\sim 2.00$ & $\sim 4.00$ & $\sim 3.00$ & 0.0665 & 0.1331 & 0.0961 \\
\hline Kuiper belt (Кв) & $\sim 30.0$ & $\sim 55.0$ & $\sim 42.0$ & 0.0048 & 0.0088 & 0.0066 \\
\hline
\end{tabular}



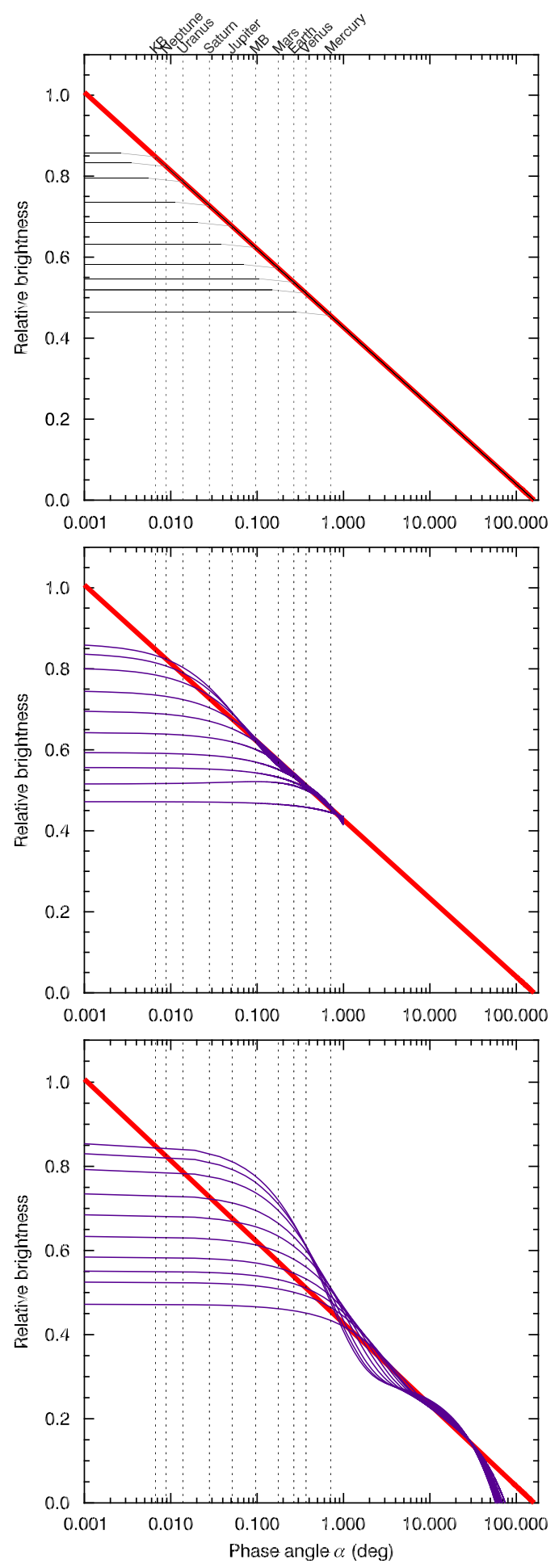

Fig. 7. Top panel: Synthetic phase curves that flatten at the apparent solar radius of the planets of the Solar System ( $\bar{\alpha}_{\odot}$ from Table 2$)$. The deconvolved data is marked in red and is the model of [14]. Second panel: Result of the best fit with the linear-exponential function at phase angle less than $1^{\circ}$. Bottom panel: Result of the best fit with the linearexponential function at phase angles from $10^{-3}$ degree to $180^{\circ}$. (For interpretation of the references to color in this figure legend, the reader is referred to the web version of this article.) angular radius of apparent solar disk seen by Mercury and that seen by Kuiper belt asteroids.

Then, we derive synthetic phase functions by creating the flattening at the apparent solar radii $\left(\bar{\alpha}_{\odot}\right.$ from Table 2$)$ seen from Mercury to Neptune. These functions assume a logarithmic increase over the full range of phase angle, which is acceptable at first order (see Fig. 8 of the Supplementary material).

We modeled the convolved synthetic phase functions with a morphological model and a convolved morphological model. The convolution model provides the expected behavior by flattening at the apparent solar radii. By contrast, the morphological model is unable to fit the synthetic data at high phase angle and the flattening at low phase angles. As shown in Fig. 7, a reasonable fit to the flattening part of the phase curves is only possible for phase angles less than $1^{\circ}$. This demonstrates that the convolution method has a major role when fitting a phase curve with a full coverage in phase angle.

\section{Discussion}

While the deconvolution method offers a solution for considering point-like sources, it is limited by its basic logarithmic shape. At the first order, it is acceptable to describe a phase curve by a logarithmic behavior (see also [97, Chap. 11B, p. 287]), but a closer look shows that observed data at phase angles greater than $1^{\circ}$ curls around the logarithmic function (see Fig. 8 of the Supplementary material). This curling behavior is also noted for the data at phase angles less than $1^{\circ}$ (see Fig. 3 ).

Moreover, as pointed out by [90], the Maxwell equations require that the derivative of the unconvolved brightness at $\alpha=0^{\circ}$ must be zero. This naturally is a rather strong challenge to the whole deconvolution problem. One possible way to solve the problem lies in the work by [98] with their RT-C model which shows an exceedingly sharp opposition peak but a zero derivative at $\alpha=0^{\circ}$. Their work points out that there exist physical models which show the required properties to explain the behavior of the opposition effect. Another way to look at the problem is to try some combination of analytical functions that have a zero derivative at $\alpha=0^{\circ}$ and a sharp opposition peak, but with a non-constant behavior at high phase angles. This could be done with the diffraction model of [56] combined with a linear function, as similarly done with the linear-exponential function of [71]. Alternative functions will be tested in a forthcoming paper.

\section{Conclusion}

To better understand the behavior of the brightness near the solar angular radius:

(1) We convolved the morphological model of [71] with solar limb darkening functions of $[59,60]$. We find a good agreement between the two convolution models. The flattening of the phase curves is progressive and effective at approximately $0.4 \alpha_{\odot}$. This value is found either by looking at the phase curves, or with 
the derivative of the convolved linear-exponential function fit to the data.

(2) We deconvolved the data by using the logarithmic function of [93]. The deconvolution model exhibits the same trend than laboratory measurements of [78].

We conclude that most of the laboratory phase curves do not exhibit similar flattening than planetary phase curves due to the difficulty of reaching phase angles as small as the angular radius of the light source (for example $\alpha_{\circledast}=0.0074^{\circ}$ for the laser beam used by [86] whereas their smallest measured phase angle was $0.025^{\circ}$ ).

To quantify the impact of the solar angular radius on the phase curves of objects located in the Solar System:

(3) We created synthetic phase functions by creating the flattening at the apparent solar radii seen from Mercury to Neptune.

(4) We modeled the convolved synthetic phase functions with a morphological model and a convolved morphological model. While the morphological model can mimic the flattening at low phase angles $\left(\alpha<1^{\circ}\right)$, it is unable to fit the synthetic data at high phase angle in the same time.

\section{Acknowledgments}

This study is performed at Jet Propulsion Laboratory (JPL), under contract with NASA and California Institute of Technology and is funded by the NASA Postdoctoral Program led by OakRidge Associated Universities (ORAU).

\section{Appendix A. Supplementary material}

Supplementary data associated with this article can be found in the online version, at doi:10.1016/j.jqsrt.2012.02. 040 .

\section{References}

[1] Gehrels T, Coffeen T, Owings D. Wavelength dependance of polarization. III. The lunar surface. Astron J 1964;69:826, doi:10. $1086 / 109359$

[2] Pohn HA, Radin HW, Wildey RL. The Moon's photometric function near zero phase angle from Apollo 8 photography. Astrophys J Lett 1969;157:L193-5.

[3] Whitaker EA. An investigation of the Lunar Heiligenschein. NASA Spec Publ 1969;201:38.

[4] Muinonen K. Coherent backscattering by solar system dust particles. In: Milani A, di Martino M, Cellino A, editors. Asteroids, comets, meteors 1993. IAU symposium, vol. 160; 1994. p. 271.

[5] Helfenstein P, Veverka J, Hillier J. The lunar opposition effect: a test of alternative models. Icarus 1997;128:2-14.

[6] Helfenstein P, Currier N, Clark BE, Veverka J, Bell M, Sullivan R, et al. Galileo observations of Europa's opposition effect. Icarus 1998;135: 41-63.

[7] Shkuratov YG, Kreslavsky MA, Ovcharenko AA, Stankevich DG, Zubko ES, Pieters C, et al. Opposition effect from Clementine data and mechanisms of backscatter. Icarus 1999;141:132-55.

[8] Belskaya IN, Shevchenko VG, Kiselev NN, Krugly YN, Shakhovskoy NM, Efimov YS, et al. Opposition polarimetry and photometry of S- and E-type asteroids. Icarus 2003;166:276-84.

[9] Verbiscer AJ, French RG, McGhee CA. The opposition surge of Enceladus: HST observations 338-1022 nm. Icarus 2005;173:66-83.
[10] Shkuratov YG, Videen G, Kreslavsky M, Belskaya I, Kaydash V, Ovcharenko A, et al. Scattering properties of planetary regoliths near opposition. In: NATO science series, vol. 161. Netherlands: Springer; 2005.

[11] Mishchenko MI, Rosenbush VK, Kiselev NN. Weak localization of electromagnetic waves and opposition phenomena exhibited by high-albedo atmosphereless solar system objects. Appl Opt 2006;45: 4459-63.

[12] Rosenbush V, Kiselev N, Avramchuk V. Similarity and diversity in photometric and polarimetric opposition effects of small Solar System bodies. J Quant Spectrosc Radiat Transfer 2006;100:325-39.

[13] Rabinowitz DL, Schaefer BE, Tourtellotte SW. The diverse solar phase curves of distant Icy bodies. I. Photometric observations of 18 Trans-Neptunian objects, 7 Centaurs, and Nereid. Astron J 2007;133: 26-43.

[14] Déau E, Dones L, Rodriguez S, Charnoz S, Brahic A. The opposition effect in the outer solar system: a comparative study of the phase function morphology. Planet Space Sci 2009;57:1282-301.

[15] Seeliger H. Zur photometrie des saturnringes. Astron Nachr 1884;109:305.

[16] Seeliger H. Zur theorie der beleuchtung der grossen planeten inbesondere des saturn. Abh Bayer Akad Wiss Math-Naturwiss Kl 1887;16:405-516.

[17] Irvine WM. The shadowing effect in diffuse reflection. J Geophys Res 1966;71:2931-7.

[18] Lumme K, Bowell E. Radiative transfer in the surfaces of atmosphereless bodies. I-theory. Astron J 1981;86:1694-721.

[19] Stankevich DG, Shkuratov YG, Muinonen K. Shadow hiding effect in inhomogeneous layered particulate media. J Quant Spectrosc Radiat Transfer 1999;63:445-8.

[20] Muinonen K, Stankevich D, Shkuratov YG, Kaasalainen S, Piironen J. Shadowing effect in clusters of opaque spherical particles. J Quant Spectrosc Radiat Transfer 2001;70:787-810.

[21] Stankevich DG, Shkuratov YG. Multiple scattering of light in regolith-like media: geometric optics approximation. Sol Syst Res 2002;36:409-16.

[22] Hapke B. Bidirectional reflectance spectroscopy. III-correction for macroscopic roughness. Icarus 1984:59:41-59.

[23] Spencer JR. A rough-surface thermophysical model for airless planets. Icarus 1990;83:27-38.

[24] Lagerros JSV. Thermal physics of asteroids. IV. Thermal infrared beaming. Astron Astrophys 1998;332:1123-32.

[25] Shepard MK, Campbell BA. Shadows on a planetary surface and implications for photometric roughness. Icarus 1998;134:279-91.

[26] Shkuratov YG, Helfenstein P. The opposition effect and the quasifractal structure of regolith: I. Theory. Icarus 2001;152:96-116.

[27] Shkuratov YG, Petrov D, Videen G. Classical photometry of prefractal surfaces. J Opt Soc Am A 2003;20(11):2081-92.

[28] Shkuratov YG, Stankevich DG, Petrov DV, Pinet PC, Cord AM, Daydou $\mathrm{YH}$, et al. Interpreting photometry of regolith-like surfaces with different topographies: shadowing and multiple scattering. Icarus 2005;173:3-15.

[29] Kuga Y, Ishimaru A. Retroreflectance from a dense distribution of spherical particles. J Opt Soc Am 1984;1:831-5.

[30] Wolf P-E, Maret G. Weak localization and coherent backscattering of photons in disordered media. Phys Rev Lett 1985;55:2696-9.

[31] Shkuratov YG. On the origin of the opposition effect and negative polarization for cosmic bodies with solid surface. Astron Circ Moscow: Sternberg State Astron Inst 1985;1400:1-16.

[32] Tsang L, Ishimaru A. Theory of backscattering enhancement of random discrete isotropic scatterers based on the summation of all ladder and cyclical terms. J Opt Soc Am A 1985;2:1331-8.

[33] Shkuratov YG, Grynko YS. Light scattering by media composed of semitransparent particles of different shapes in ray optics approximation: consequences for spectroscopy, photometry, and polarimetry of planetary regoliths. Icarus 2005;173:16-28.

[34] Stankevich D, Shkuratov YG, Grynko Y, Muinonen K. Computer simulations for multiple scattering of light rays in systems of opaque particles. J Quant Spectrosc Radiat Transfer 2003;76:1-16.

[35] Stankevich D, Shkuratov YG. Monte carlo ray-tracing simulation of light scattering in particulate media with optically contrast structure. J Quant Spectrosc Radiat Transfer 2004;87:289-96.

[36] Shkuratov YG. A diffraction mechanism for the formation of the opposition effect of the brightness of surfaces having a complex structure. Kinematika i Fiz Nebesnykh Tel 1988;4:33-9.

[37] Muinonen K. Light scattering by inhomogeneous media: backward enhancement and reversal of linear polarization, $\mathrm{PhD}$ thesis. Finland: University of Helsinki; 1990. 
[38] Hapke B. Coherent backscatter and the radar characteristics of outer planet satellites. Icarus 1990;88:407-17.

[39] Mishchenko MI. The angular width of the coherent back-scatter opposition effect-an application to icy outer planet satellites. Astrophys Space Sci 1992;194:327-33.

[40] Petrova EV, Tishkovets VP, Jockers K. Modeling of opposition effects with ensembles of clusters: interplay of various scattering mechanisms. Icarus 2007;188:233-45.

[41] Tishkovets VP, Shrukratov YG, Litvinov PV. Comparison of collective effects at scattering by randomly oriented clusters of spherical particles. J Quant Spectrosc Radiat Transfer 1999;61:767-73.

[42] Zubko E, Shkuratov YG, Muinonen K, Videen G. Collective effects by agglomerated debris particles in the backscatter. J Quant Spectrosc Radiat Transfer 2006;100:489-95.

[43] Muinonen K, Zubko E, Tyynelä J, Shkuratov YG, Videen G. Light scattering by Gaussian random particles with discrete-dipole approximation. J Quant Spectrosc Radiat Transfer 2007;106:360-77.

[44] Tyynelä J, Zubko E, Videen G, Muinonen K. Interrelating angular scattering characteristics to internal electric fields for wavelengthscale spherical particles. J Quant Spectrosc Radiat Transfer 2007;106: 520-34.

[45] Shkuratov YG, Bondarenko S, Ovcharenko A, Pieters C, Hiroi T, Volten $\mathrm{H}$, et al. Comparative studies of the reflectance and degree of linear polarization of particulate surfaces and independently scattering particles. J Quant Spectrosc Radiat Transfer 2006;100: 340-58.

[46] Shkuratov YG, Bondarenko S, Kaydash V, Videen G, Muñoz O, Volten $\mathrm{H}$. Photometry and polarimetry of particulate surfaces and aerosol particles over a wide range of phase angles. J Quant Spectrosc Radiat Transfer 2007;106:487-508.

[47] Muñoz O, Volten H, Hovenier JW, Min M, Shkuratov YG, Jalava JP, et al. Experimental and computational study of light scattering by irregular particles with extreme refractive indices: hematite and rutile. Astron Astrophys 2006;446(2):525-35

[48] Hapke B. The Lumme-Bowell photometric parameters: reality or fantasy?Bull Am Astron Soc 1982;14(June):726-7

[49] Lumme K, Bowell E. A reply to Hapke's criticism of the LummeBowell photometric theory. Bull Am Astron Soc 1982;14(June): 726-7.

[50] Lumme K, Bowell E. A note on Hapke's bidirectional reflectance spectroscopy. Part 3: correction for macroscopic roughness. NASA STI/Recon Technical Report No. 86: 29739; 1986.

[51] Shkuratov YG, Zubko ES. Comment on modeling of opposition effects with ensembles of clusters: interplay of various scattering mechanisms by Elena V. Petrova, Victor P. Tishkovets, Klaus Jockers, 2007 [Icarus 188, 233 245]. Icarus 2008;194(April): 850-2

[52] Petrova EV, Tishkovets VP, Jockers K. Rebuttal to comment on Modeling of opposition effects with ensembles of clusters: interplay of various scattering mechanisms by Elena V. Petrova, Victor P. Tishkovets, Klaus Jockers, 2007 [Icarus 188, 233 245]. Icarus 2008;194(April):853-6.

[53] Hapke BW, Nelson RM. Comments on Approximate calculation of coherent backscattering for semi-infinite discrete random media by Victor P. Tishkovets, and Michael I. Mishchenko. J Quant Spectrosc Radiat Transfer 2010;111:643-4.

[54] Tishkovets VP, Mishchenko MI. Coherent backscattering: conceptions and misconceptions (reply to comments by Bruce W. Hapke and Robert M. Nelson). J Quant Spectrosc Radiat Transfer 2010;111: 645-9.

[55] Kawata Y, Irvine WM. Models of Saturn's rings which satisfy the optical observations. In: Woszczyk A, Iwaniszewska C, editors Exploration of the planetary system. IAU symposium, vol. 65; 1974. p. 441-64.

[56] Shkuratov YG. Estimating the effect of finite angular light source dimensions on the opposition brightness effect in atmosphereless bodies. Sol Syst Res 1991;25:54.

[57] Nozette S, Rustan P, Pleasance LP, Horan DM, Regeon P, Shoemake EM, et al. The clementine mission to the moon: scientific overview. Science 1994;266:1835-9.

[58] Y.G. Shkuratov, D.G. Stankevich, Can lunar opposition spike measured by Clementine exist? Lunar and Planetary Institute Science Conference Abstracts, vol. 26. Technical Report. Lunar and Planetary Institute; March 1995. p. 1295.

[59] Pierce AK, Waddell JH. Analysis of limb darkening observations. Mem R Astron Soc 1961;63:89-112.

[60] Hestroffer D, Magnan C. Wavelength dependency of the Solar limb darkening. Astron Astrophys 1998;333:338-42.
[61] Mishchenko MI, Dlugach JM, Liu L, Rosenbush VK, Kiselev NN Shkuratov YG. Direct solutions of the Maxwell equations explain opposition phenomena observed for high-albedo solar system objects. Astrophys J Lett 2009;705:L118-22.

[62] Helfenstein P, Veverka J. Photometric properties of lunar terrains derived from Hapke's equation. Icarus 1987;72:342-57.

[63] Domingue DL, Hapke BW, Lockwood GW, Thompson DT. Europa's phase curve-implications for surface structure. Icarus 1991;90: 30-42.

[64] Poulet F, Cuzzi JN, French RG, Dones L. A study of saturn's ring phase curves from HST observations. Icarus 2002;158:224-48.

[65] Déau E, Charnoz S, Dones L, Brahic A, Porco CC. ISS/Cassini observes the opposition effect in Saturn's rings: I. Morphology of optical phase curves, Icarus, submitted for publication [under final review, 2012].

[66] Murray CD, Dermott SF. Solar system dynamics, ISBN 0521575974 UK: Cambridge University Press; February 2000.

[67] Belton MJS, Head III JW, Ingersoll AP, Greeley R, McEwen AS, Klaasen KP, et al. Galileo's first images of Jupiter and the Galilean satellites. Science 1996;274:377-85

[68] Nelson RM, Hapke BW, Brown RH, Spilker LJ, Smythe WD, Kamp L, et al. Cassini observations of the opposition effect of Saturn's rings1. In: Mackwell S, Stansbery E, editors. 37th annual lunar and planetary science conference, vol. 47. Technical Report. Lunar and Planetary Institute; 2006. p. 1461.

[69] Nelson R. Laboratory investigations relevant to Cassini VIMS reports of coherent constructive interference in Saturn's Rings. 37th COSPAR scientific assembly of COSPAR, Plenary Meeting, vol. 37; 2008. p. 2200

[70] Muinonen K, Shkuratov YG, Ovcharenko A, Piironen J, Stankevich D Miloslavskaya O, et al. The SMART-1 AMIE experiment: implication to the lunar opposition effect. Planet Space Sci 2002;50:1339-44.

[71] Kaasalainen S, Muinonen K, Piironen J. Comparative study on opposition effect of icy solar system objects. J Quant Spectrosc Radiat Transfer 2001;70:529-43.

[72] French RG, Verbiscer A, Salo H, McGhee C, Dones L. Saturn's rings at true opposition. Publ Astron Soc Pac 2007;119:623-42.

[73] Salo H, French RG. The opposition and tilt effects of Saturn's rings from HST observations. Icarus 2010;210:785-816.

[74] Déau E, Brahic A, Dones L, Porco CC. Benchmark of convolution and deconvolution models: implications for planetary opposition surges. In: Muinonen K, Penttilä A, Lindqvist $H$, Nousiainen $T$, Videen G. editors. Electromagnetic and light scattering XII; June 2010. p. 30

[75] Thuillier G, Claudel J, Djafer D, Haberreiter M, Mein N, Melo SML et al. The shape of the solar limb: models and observations Sol Phys 2011;268:125-49.

[76] Etemad S, Thompson R, Andrejco MJ, John S, Mackintosh FC. Weak localization of photons: termination of coherent random walks by absorption and confined geometry. Phys Rev Lett 1987;59:1420-3.

[77] Tishkovets VP, Mishchenko MI. Approximate calculation of coherent backscattering for semi-infinite discrete random media. J Quant Spectrosc Radiat Transfer 2009;110:139-45.

[78] Psarev V, Ovcharenko A, Shkuratov YG, Belskaya I, Videen G Photometry of particulate surfaces at extremely small phase angles. J Quant Spectrosc Radiat Transfer 2007;106:455-63.

[79] Capaccioni F, Cerroni P, Barucci MA, Fulchignoni M. Phase curves of meteorites and terrestrial rocks-laboratory measurements and applications to asteroids. Icarus 1990;83:325-48

[80] Nelson RM, Hapke BW, Smythe WD, Horn LJ. Phase curves of selected particulate materials: the contribution of coherent backscattering to the opposition surge. Icarus 1998;131:223-30.

[81] Nelson RM, Hapke BW, Smythe WD, Spilker LJ. The opposition effect in simulated planetary regoliths, reflectance and circular polarization ratio change at small phase angle. Icarus 2000;147: 545-58.

[82] Kaasalainen S, Piironen J, Muinonen K, Karttunen H, Peltoniemi J. Laboratory experiments on backscattering from regolith samples. Appl Opt 2002;41:4416-20.

[83] Kaasalainen S. Laboratory photometry of planetary regolith analogs. I. Effects of grain and packing properties on opposition effect. Astron Astrophys 2003;409:765-9.

[84] Sakai T, Tomita N, Nakamura AM. Bidirectional reflectance of asteroid surface analogues: quantification of porosity and surface roughness. In: Mackwell S, Stansbery E, editors. Lunar and planetary institute science conference abstracts, vol. 34; 2003. p. 1618.

[85] Näränen J, Kaasalainen S, Peltoniemi J, Heikkilä S, Granvik M, Saarinen V. Laboratory photometry of planetary regolith analogs. 
II. Surface roughness and extremes of packing density. Astron Astrophys 2004;426:1103-9.

[86] Piatek JL, Hapke BW, Nelson RM, Smythe WD, Hale AS. Scattering properties of planetary regolith analogs. Icarus 2004;171:531-45.

[87] Sakai T, Nakamura AM. Quantication of porosity and surface roughness in laboratory measurements of the bidirectional reflectance of asteroid surface analogues. Earth Planets Space 2005;57:71-6.

[88] Kawakami K, Nakamura AM. Near infrared opposition surge of carbonaceous chondrite meteorites. In: Lunar and planetary institute science conference abstracts, vol. 38; March 2007. p. 1531.

[89] Hapke BW, Shepard MK, Nelson RM, Smythe WD, Piatek JL. A quantitative test of the ability of models based on the equation of radiative transfer to predict the bidirectional reflectance of a wellcharacterized medium. Icarus 2009;199:210-8.

[90] Shkuratov YG, Psarev V, Stankevich D, Ovcharenko A, Videen G. Why the opposition spikes of regolith-like media are usually sharp and do not show rounding off. In: Muinonen $\mathrm{K}$, Penttilä $\mathrm{A}$, Lindqvist $\mathrm{H}$, Nousiainen T, Videen G, editors. Electromagnetic and light scattering XII. Proceedings of the 12th conference held in Helsinki, June 28-July 2, 2010. Helsinki: Helsinki University Print; 2010. p. 278.

[91] Shkuratov YG, Ovcharenko A, Zubko E, Miloslavskaya O, Muinonen K, Piironen J, et al. The opposition effect and negative polarization of structural analogs for planetary regoliths. Icarus 2002;159:396-416.
[92] Nakamura AM, Kamei A, Kogachi M, Mukai T. Laboratory measurements of laser-scattered light by rough surfaces. Adv Space Res 1999;23:1201-4.

[93] Bobrov MS. Physical properties of Saturn's rings. In: Dollfus A, editor. Surfaces and interiors of planets and satellites. New York: Academic; 1970. p. 376-461.

[94] Shkuratov YG. The effect of the finiteness of the angular dimensions of a light source on the magnitude of the opposition effect of the luminosity of atmosphereless bodies. Astron Vestn 1991;25:71-5.

[95] Hapke B. Bidirectional reflectance spectroscopy V. The coherent backscatter opposition effect and anisotropic scattering. Icarus 2002;157:523-34.

[96] Mallama A, Wang D, Howard RA. Photometry of mercury from SOHO/LASCO and earth, the phase function from 2 to $170 \mathrm{deg}$. Icarus 2002;155:253-64.

[97] Hapke B. Theory of reflectance and emittance spectroscopy. In: Topics in remote sensing. Cambridge, UK: Cambridge University Press; 1993.

[98] Muinonen K, Zubko E. Coherent backscattering by a finite medium of particles. In: Muinonen K, Penttilä A, Lindqvist H, Nousiainen T, Videen G., editors. Electromagnetic and light scattering XII; June 2010. p. 194. 